\title{
Diversité et Structure du Peuplement Ichtyologique du Bassin Inférieur du Fleuve Comoé (Côte d'Ivoire)
}

\author{
Yao Abissa Antoine, \\ Konan Koffi Mexmin, \\ Doumbia Lassina, \\ Ouattara Allassane, \\ Gourene Germain,
} Laboratoire d'Environnement et de Biologie Aquatique, Université Nangui Abrogoua, Abidjan, Côte d'Ivoire

Doi: 10.19044/esj.2019.v15n6p244 URL:http://dx.doi.org/10.19044/esj.2019.v15n6p244

\section{Résumé}

La présente étude vise à évaluer la diversité des poissons dans le bassin inférieur du fleuve Comoé. De façon spécifique il s'agit de faire l'inventaire ichtyologique de ce milieu, d'identifier les facteurs déterminant la diversité ichtyologique de ce fleuve. Les données sont issues des pêches expérimentales et artisanales durant huit campagnes saisonnières, de juin 2014 à mai 2017. Cette étude a permis d'identifier 67 espèces de poissons reparties en 11 ordres et 30 familles. Parmi ces 67 espèces 16 sont signalées pour la première fois dans le bassin du fleuve. Les Perciformes $(40,30 \%)$, Siluriformes $(17,91 \%)$ et Osteoglossiformes $(11,94 \%)$ sont les plus représentés. En termes d'abondance, sur 7265 spécimens capturés, la plus forte valeur (2428) soit 33,42 \% a été enregistrée à Adjékro et la plus faible (583) soit 8,02\% à Moossou. Du point de vue de la distribution, les valeurs de l'indice de Shannon-Weaver (H') et l'équitabilité (E) montrent que les peuplements ichtyologiques sont équilibrés dans l'ensemble. Les paramètres liés à la minéralisation ( $\mathrm{pH}$ et conductivité) et la transparence apparaissent les plus discriminants dans la répartition des poissons dans les eaux.

Mots-clés: Diversité de poissons, structure du peuplement, bassin inférieur, fleuve Comoé, Côte d'Ivoire 


\title{
Diversity and Structure of the Ichtyological Settlement of the Lower Basin of the Comoé River (Côte d'Ivoire)
}

\author{
Yao Abissa Antoine, \\ Konan Koffi Mexmin, \\ Doumbia Lassina, \\ Ouattara Allassane, \\ Gourene Germain,
}

Laboratoire d'Environnement et de Biologie Aquatique, Université Nangui Abrogoua, Abidjan, Côte d'Ivoire

\begin{abstract}
The present study aims to assess the diversity of fish in the lower basin of the Comoé River. In a specific manner, it comes to doing survey, to identify the factors determining the ichtyological diversity of this river. Data presented are from experimental and artisanal fisheries during eight seasonal campaigns, from June 2014 to May 2017. This study identified 67 fish species divided into 11 orders and 30 families. Of these 67 species, 16 are reported for the first time in the river basin. Perciformes $(40.30 \%)$, Siluriformes $(17.91 \%)$ and Osteoglossiformes (11.94\%) are the most represented. In terms of abundance, the highest value, that is, 2428 out of 7265 specimens captured, or $33.42 \%$, was recorded at Adjékro and the lowest, that is 583, or $8.02 \%$ at Moossou. From a distribution point of view, the Shannon-Weaver $(\mathrm{H} ')$ index and equitability (E) values indicate that the fish populations are generally balanced. The parameters related to mineralization ( $\mathrm{pH}$ and conductivity) and transparency appear to be the most discriminating in the distribution of fish in the waters.
\end{abstract}

Keywords: Fish diversity, stand structure, lower basin, Comoé River, Côte d'Ivoire

\section{Introduction}

La pêche, activité très ancienne de prélèvement sur le milieu naturel, est un bon exemple de la manière dont les hommes pensent et mettent en œuvre leur rapport avec l'environnement (Paugy et al., 2011). La grande diversité des engins de pêche et des techniques révèle aussi que les pêcheurs 
connaissent des particularités écologiques et biologiques des poissons. Les techniques de capture, très primitives à l'origine, ont évolué pour pouvoir capturer une plus grande variété d'espèces dans différents types de milieux aquatiques. Des études anthropologiques contemporaines sur les sociétés primitives ont confirmé l'importance de la pêche comme moyen de subsistance (Arnason, 1997, Ahouanssou, 2011). Selon Arnason (1997), des colonies humaines sont souvent établies dans des régions où les prises de poissons sont relativement bonnes. Une portion du cours inférieur du fleuve Comoé faisant objet d'étude, est incluse dans zone humide de Grand Bassam classée "Site RAMSAR d'importance internationale" (Ramsar Iran, 1971 ; Secrétariat de la Convention de Ramsar, 2013 ; Ramsar, 2005). Cette zone est un site d'importance écologique du fait de la diversité des habitats et de la diversité biologique qu'elle renferme. Malgré son statut de protection, elle subit une forte pression anthropique ayant pour conséquence sa destruction et une menace des espèces aquatiques qui y vivent. Ces menaces sont de tout ordre notamment les pollutions chimiques, microbiologiques, mais surtout l'exploitation par la pêche abusive et non contrôlée des ressources halieutiques. En dehors de la pêche, certaines activités anthropiques affectent directement ou indirectement les ressources halieutiques. Ces ressources biologiques aquatiques sont donc menacées par l'utilisation de produits phytosanitaires en agriculture, le rejet de déchets ménagers et l'insuffisance de textes réglementaires en matière de gestion des écosystèmes aquatiques. Pour Charles-Dominique (2008), un bien commun en accès libre est voué à la dégradation par le jeu de la compétition entre exploitants, qui cherchent à maximiser leur intérêt individuel sauf si une autorité centrale n'intervient. L'expérience a montré que les systèmes d'accès libre, aboutissent systématiquement à une surexploitation des ressources et à une baisse des recettes pour les participants (Traoré, 1996). Par ailleurs, Vanga et al. (2002) indiquent qu'une pêche trop intensive entraine une moindre durée de vie du poisson, une taille réduite des individus débarqués, une diminution de l'abondance, des rendements, de la rentabilité des opérations de pêche et souvent, une modification de la composition des captures au profit d'espèces moins prisées. Pour pallier ce problème Gascuel (2009) propose l'approche écosystémique des pêches comme une application des principes du développement durable au domaine de l'exploitation des ressources et écosystèmes. Elle a pour but de planifier, de valoriser et de gérer les pêches. Cette démarche place, en effet, les activités humaines au centre de l'écosystème. Elle vise à préserver la biodiversité et à minimiser les impacts des activités humaines sur les écosystèmes. L'état des peuplements piscicoles constitue un des éléments de qualité permettant l'évaluation de l'état écologique des cours d'eau. Outre la qualité physico-chimique de l'eau, les poissons sont sensibles au régime hydrologique et à l'état physique des cours 
d'eau. Il apparaît nécessaire d'inventorier la faune ichtyologique de cette partie de ce cours d'eau en vue de l'établissement d'un référentiel ichthyen dans le cadre de la mise en pratique d'une Approche Ecosystémique des Pêches (AEP). En absence d'informations factuelles et de données scientifiques récentes, la présente étude réalisée sur le bassin inférieur du fleuve Comoé vise à inventorier les espèces de poissons et à déterminer la structure du peuplement à partir des captures opérées par la pêche artisanale et la pêche expérimentale.

\section{Matériel et Méthodes \\ Milieu d'étude}

Six (6) sites dont cinq (5) sur le lit principal et un (1) sur l'affluent appelé "Lagune de Kodjoboué"' ont été retenus dans le bassin inférieur du fleuve Comoé (Figure 1). Ils ont été choisis en tenant compte de leur accessibilité, des activités de pêche et des activités anthropiques qui s'y déroulent. Il s’agit de Motobé, Yaou, Palm-CI, Adjékro et Moossou. Les activités menées autour des différentes stations sont indiquées dans le tableau 1. On observe à tous les sites la présence de végétaux aquatiques envahissants à la surface de l'eau.

Tableau 1 : Caractéristiques des sites d'échantillonnage sur le bassin inférieur du fleuve Comoé

\begin{tabular}{|c|c|c|c|}
\hline Stations & Occupations des sols & Substrat $(\%)$ & Canopée (\%) \\
\hline Motobé & $\begin{array}{l}\text { Village, plantations de cacao, d'hévéa et } \\
\text { de banane }\end{array}$ & Boue (45\%), argile-limon (50\%) & 5 \\
\hline Yaou & $\begin{array}{l}\text { Village, dépôt d'ordures ménagères, } \\
\text { plantations de cacao et d'hévéa }\end{array}$ & $\begin{array}{l}\text { Sable }(15 \%) \text {, argile-limon (25\%), } \\
\text { boue }(60 \%)\end{array}$ & 0 \\
\hline Palm-CI & $\begin{array}{l}\text { Campement, pépinières de palmier à } \\
\text { huile et plantation de cacao }\end{array}$ & Boue $(80 \%)$, sable $(20 \%)$ & 0 \\
\hline Adjékro & $\begin{array}{l}\text { Campement, unité de traitement de fibres } \\
\text { de coco, plantations d'hévéa et de } \\
\text { cocotiers }\end{array}$ & $\begin{array}{l}\text { Sable }(15 \%) \text {, boue }(50 \%) \text {, argile- } \\
\text { limon }(35 \%)\end{array}$ & 0 \\
\hline Moossou & Pont, cultures maraichères & Boue $(80 \%)$, argile-limon $(20 \%)$ & 0 \\
\hline Kodjoboué & $\begin{array}{l}\text { Village, peu d'activités anthropiques, } \\
\text { quelques champs en bordure }\end{array}$ & Sable, macrophytes (5\%) & 0 \\
\hline
\end{tabular}

\section{Collecte des données}

Les paramètres physiques et chimiques ont été mesurés entre 8 heures et 12 heures aux différentes stations choisies. Les mesures de température, $\mathrm{pH}$, conductivité, oxygène dissous, transparence et profondeur ont été effectuées in situ. Les quatre premiers paramètres cités ont été mesurés à l'aide d'un multiparamètre HANNA Hi 9828. La transparence des eaux a été déterminée avec un disque de Secchi. La profondeur de l'eau a été évaluée à l'aide d'une corde graduée et lestée. Le couvert forestier (en \%), le type de substrat (boue, sable, gravier) et des débris ligneux (en \%) ont été estimés visuellement à 
chaque site d'échantillonnage comme décrit par Gordon et al. (1994) et Rios $\&$ Bailey (2006).

Les échantillonnages, basés sur des pêches expérimentales et artisanales, ont été effectués durant huit (8) campagnes saisonnières, de juin 2014 à mai 2017 (quatre (4) en saison pluvieuse et quatre (4) en saison sèche). La pêche expérimentale a été réalisée avec une batterie de 10 filets maillants de $6,10,13,15,18,20,25,30,36$ et $60 \mathrm{~mm}$ nœud à nœud, de 25 à $30 \mathrm{~m}$ de longueur et de $1,5 \mathrm{~m}$ de hauteur de chute chacun. Les filets ont été posés une seule fois par campagne dans chaque site l'après-midi entre 16 heures et 17 heures et relevés le lendemain entre 7 heures et 8 heures. Les poissons capturés ont été identifiés à l'aide des clés de Paugy et al. (2003a ; 2003b).

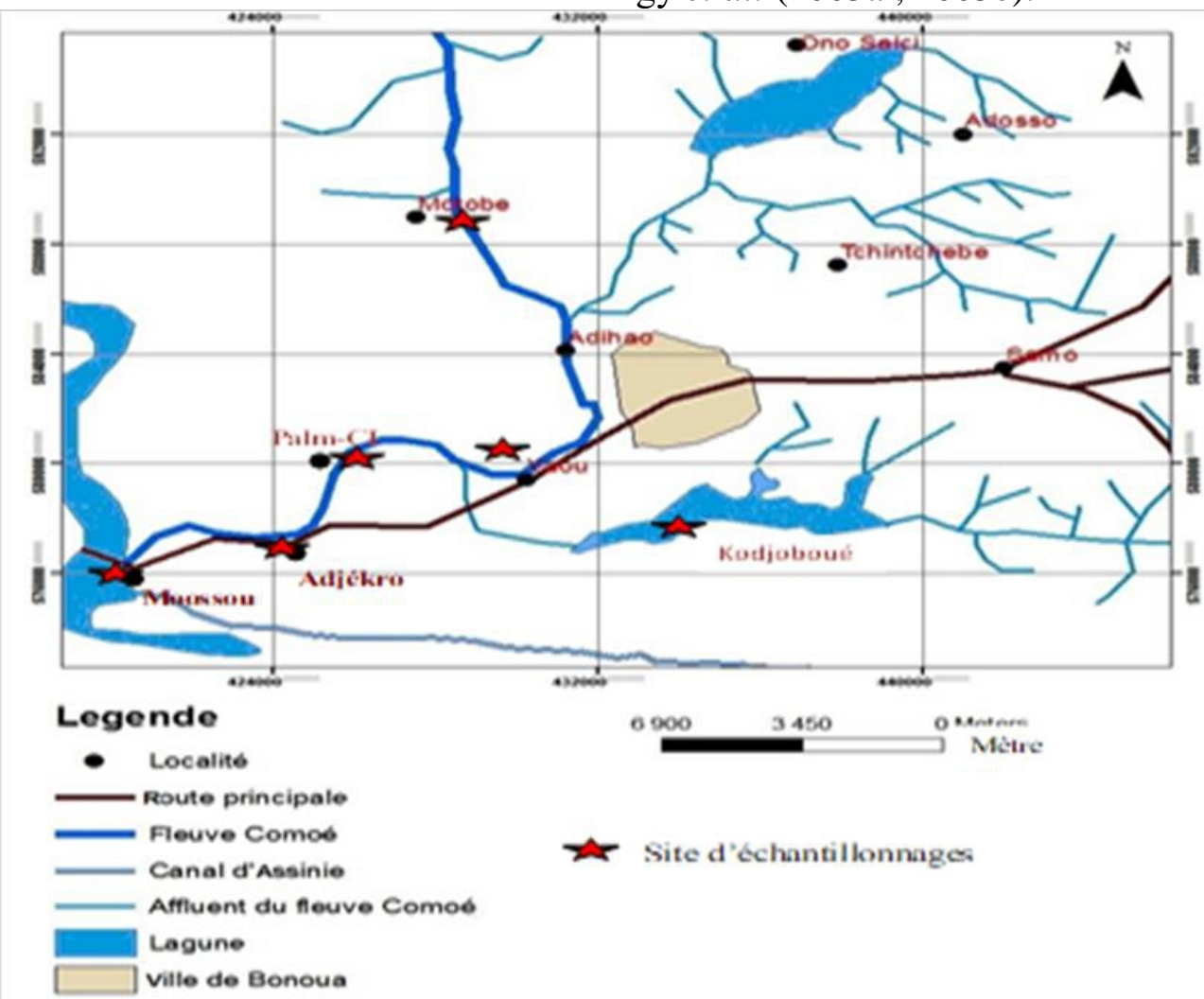

Figure 1 : Localisation des stations d'échantillonnages dans le bassin inférieur du fleuve Comoé

\section{Analyse des données}

Le peuplement ichtyologique prélevé a été caractérisé par différents types d'indices notamment, la richesse spécifique, la richesse raréfiée, l'indice de Shannon-Weaver, l'équitabilité, le pourcentage d'occurrence, l'abondance et l'indice de similarité de Jaccard. 
L'indice de Shannon (Shannon, 1948 ; Shannon \& Weaver, 1963) aussi appelé indice de Shannon-Weaver ou Shannon-Wiener (Marcon, 2015) est utilisé pour mesurer le degré d'organisation du peuplement.-Il a été calculé selon l'équation :

$$
H^{\prime}=-\sum_{i=1}^{s} p_{i}\left(\log _{2} p_{i}\right)
$$

$\mathrm{H}^{\prime}=$ Indice de diversité de Shannon-Weaver, $\mathrm{S}=$ nombre d'espèces (taxons), $p i=$ proportion par espèce $i$ (taxon).

$\mathrm{H}^{\prime}$ tend vers 0 quand la diversité est minimale. Elle est maximale quand $\mathrm{H}^{\prime}$ tend vers l'infini.

L'indice de régularité ou d'équitabilité de Pielou (1966) a permis d'apprécier la qualité de la répartition des individus au sein des espèces du milieu. Il se calcule à l'aide de la formule $: \mathrm{E}=\mathrm{H}^{\prime} / \log _{2} \mathrm{R}_{\mathrm{S}}$ où $\mathrm{E}=$ indice d'équitabilité ; $H^{\prime}=$ indice de Shannon-Weaver $; R_{\mathrm{s}}=$ nombre d'espèces.

La valeur de $\mathrm{E}$ est comprise entre 0 et 1 . Elle tend vers 0 quand la quasi-totalité des effectifs des individus est concentrée sur une espèce, et vers 1 lorsque toutes les espèces ont la même abondance.

La richesse en taxons a été raréfiée pour éviter tout biais lié aux différences d'abondance entre les échantillons (Paillex et al., 2007 ; Edia et al., 2016). Dans la présente étude, la raréfaction a été appliquée à la richesse taxonomique par échantillon en utilisant la plus faible abondance (237 individus) enregistrée dans les échantillons comme nombre cible d'individus (Okasanen et al., 2013). Les calculs ont été réalisés en utilisant le package « Vegan » (Okasanen et al., 2013) du logiciel R.

Le pourcentage d'occurrence $(\mathrm{F})$ renseigne sur les préférences de milieu (habitat) d'une espèce donnée. Il s'obtient selon la formule $F=\left(F_{i}\right.$ * 100) / F

Selon la valeur de F, la classification suivante est adoptée (Dajoz, $2000)$ : espèces constantes $(F \geq 50 \%)$, espèces accessoires $(25 \leq F \leq 50 \%)$ et espèces accidentelles $(\mathrm{F}<25 \%)$.

L'indice de similarité de Jaccard, (1908) a été utilisé pour comparer les sites deux à deux, car il évalue la ressemblance entre deux relevés en faisant le rapport entre les espèces communes aux deux relevés et celles propres à chaque relevé. Il a pour formule $C_{J}=j /(a+b-j)$ avec $J=$ nombre d'espèces communes aux deux stations, $\mathrm{a}=$ le nombre de taxons propres à la station $1, \mathrm{~b}$ $=$ le nombre de taxons propres à la station 2 et $\mathrm{CJ}=$ indice de similarité de Jaccard.

On estime que deux groupes sont semblables si la valeur est supérieure à 0,5 et dissemblables cette valeur est inférieure à 0,5 .

La régression multiple pas à pas ascendante a été utilisée pour déterminer les paramètres physiques et chimiques qui influencent 
significativement la distribution des taxons. Cette analyse s'effectue sur une variable dépendante continue en fonction de prédicteur continu. Pour un taxon donné, l'influence des prédicteurs sur le taxon considéré est significative lorsque la valeur de $p$ assiociée au coefficient de régression est inférieure ou égale à 0,05 . Les valeurs de $t$ (coefficient de régression) et de $p$ (probabilité) donnent une bonne indication de l'impact de la variable prédictive sur la variable dépendante. Une valeur absolue élevée de $t$ et une faible valeur de $p$ suggèrent que le paramètre concerné a un fort impact sur les densités des poissons. Ce test a été effectué avec le logiciel STATISTICA 7.1 (Statsoft, 2005). Selon les exigences du logiciel, toutes les espèces ayant apparu une seule fois, quelle que soit leur abondance, ont été rejetées.

\section{Résultats}

\section{Caractéristiques du milieu}

La caractérisation physique et chimique des eaux du milieu d'étude a été réalisée à partir de l'analyse des variations spatio-temporelles de la température de l'eau, $\mathrm{pH}$, oxygène dissous, conductivité et de la transparence. Les valeurs médianes de ces paramètres sont présentées dans le tableau 2. Pour les variations spatio-temporelles, l'analyse a pris en compte toutes les valeurs mesurées lors des huit campagnes d'échantillonnage. Les valeurs extrêmes de température $\left(\min =26,2^{\circ} \mathrm{C}\right.$ et $\left.\max =33^{\circ} \mathrm{C}\right)$ ont été enregistrées à la station de Motobé. La concentration minimale $(1,14 \mathrm{mg} / \mathrm{L})$ d'oxygène dissous a été obtenue à Kodjoboué et la maximale (11,50 mg/L) à Palm-CI. Le pH des eaux est compris entre 5,3 et 8,01. Ces valeurs du potentiel d'hydrogène ont été mesurées à Kodjoboué. La station de Kodjoboué a présenté la valeur minimale de conductivité qui est de $19 \mu \mathrm{S} / \mathrm{cm}$ tandis que Moossou enregistre la valeur maximale qui est $127 \mu \mathrm{S} / \mathrm{cm}$. La transparence des eaux oscille entre $8 \mathrm{~cm}$ à Yaou et 109,5 cm à Kodjoboué. Le test de Kruskal-Wallis indique que seules la conductivité $(H=22,14 ; \mathrm{dl}=5 ; \mathrm{p}=0,000)$ et la transparence $(\mathrm{H}=16,63$; $\mathrm{dl}=5 ; \mathrm{p}=0,005)$ présentent une variation spatiale significative.

Les variations saisonnières des paramètres physiques et chimiques dans l'ensemble du bassin du cours inférieur du fleuve Comoé montrent que les températures extrêmes enregistrées durant la période d'échantillonnage $\left(\min =27^{\circ} \mathrm{C}\right.$ et $\left.\max =33^{\circ} \mathrm{C}\right)$ ont été obtenues en saison sèche. Contrairement à la température, les valeurs extrêmes $(\mathrm{min}=3,45 \mathrm{mg} / \mathrm{L}$ et $\max =9,50 \mathrm{mg} / \mathrm{L})$ de l'oxygène dissous ont été notées en période de saison pluvieuse. Concernant la conductivité, le $\mathrm{pH}$, la transparence, les valeurs minimales sont obtenues en saison sèche tandis que les maximales ont été enregistrées en période pluvieuse. On constate une tendance inverse pour la transparence avec le minimum en saison de pluies. Le test U de Mann-Whitney montre qu'à la station de Motobé seule la température présente des variations saisonnières 
significatives $(\mathrm{p}<0,05)$. Les plus grandes valeurs de température ont été obtenues en saison sèche dans chacune des autres stations.

Tableau 2: Valeurs médianes des paramètres physico-chimiques mesurés dans le bassin inférieur du fleuve Comoé. $\mathbf{S S}=$ saison sèche, $\mathbf{S P}=$ saison pluvieuse, $\mathbf{T}=$ température ;

$\mathbf{C N D}=$ conductivité, Transp. $=$ transparence, $\mathbf{O}_{2}=$ oxygène dissous

\begin{tabular}{|c|c|c|c|c|c|c|}
\hline Stations & Saisons & $\mathbf{T}\left({ }^{\circ} \mathbf{C}\right)$ & $\mathrm{CND}(\mu \mathrm{S} / \mathrm{cm})$ & pH & Transp. $(\mathrm{cm})$ & $\mathrm{O}_{2}(\mathrm{mg} / \mathrm{L})$ \\
\hline \multirow{3}{*}{ Adjékro } & SS & $29,24 \pm 1,75$ & $70,50 \pm 24,36$ & $7,24 \pm 0,42$ & $56 \pm 45,30$ & $2,69 \pm 2,62$ \\
\hline & SP & $27,50 \pm 0,74$ & $78,03 \pm 23,10$ & $7,07 \pm 0,19$ & $26,50 \pm 15,42$ & $5,20 \pm 0,59$ \\
\hline & Ensemble & $28,48 \pm 1,67$ & $78,03 \pm 24,67$ & $7,11 \pm 0,30$ & $35 \pm 37,62$ & $4,60 \pm 1,97$ \\
\hline \multirow{3}{*}{ Yaou } & SS & $30,33 \pm 1,77$ & $63 \pm 21,65$ & $6,75 \pm 0,23$ & $63,50 \pm 45,74$ & $3,65 \pm 2,43$ \\
\hline & SP & $27,25 \pm 0,91$ & $83,41 \pm 24,26$ & $7,10 \pm 0,34$ & $27,50 \pm 17,38$ & $5,17 \pm 1,20$ \\
\hline & Ensemble & $28,66 \pm 1,96$ & $76,41 \pm 26,01$ & $6,85 \pm 0,30$ & $32,5 \pm 37,37$ & $4,39 \pm 1,81$ \\
\hline \multirow{3}{*}{ Motobé } & SS & $30,50 \pm 1,52$ & $68 \pm 23,51$ & $6,89 \pm 0,34$ & $45 \pm 27,65$ & $5,74 \pm 2,53$ \\
\hline & SP & $27,50 \pm 1,13$ & $79,90 \pm 31,47$ & $7,07 \pm 0,33$ & $25 \pm 7,93$ & $6,31 \pm 1,43$ \\
\hline & Ensemble & $28,93 \pm 1,98$ & $77,53 \pm 27,61$ & $7,07 \pm 0,34$ & $27,50 \pm 22,76$ & $6,24 \pm 2,17$ \\
\hline \multirow{3}{*}{ Moossou } & SS & $29,25 \pm 1,21$ & $97 \pm 40,16$ & $7,18 \pm 0,57$ & $109,50 \pm 48,39$ & $1,80 \pm 2,75$ \\
\hline & SP & $27,45 \pm 0,71$ & $85,22 \pm 38,06$ & $6,70 \pm 0,52$ & $24 \pm 23,11$ & $5,75 \pm 2,31$ \\
\hline & Ensemble & $28,35 \pm 1,37$ & $85,22 \pm 36,31$ & $7,02 \pm 0,51$ & $46,57 \pm 49,47$ & $4,26 \pm 2,92$ \\
\hline \multirow{3}{*}{ Palm-CI } & SS & $30,12 \pm 1,40$ & $64,50 \pm 20,50$ & $7,12 \pm 0,50$ & $54 \pm 33,64$ & $1,57 \pm 1,38$ \\
\hline & SP & $27,55 \pm 0,98$ & $69,91 \pm 23,50$ & $6,84 \pm 0,41$ & $28 \pm 11,74$ & $8,53 \pm 2,79$ \\
\hline & Ensemble & $29,01 \pm 1,73$ & $69,91 \pm 23,03$ & $6,89 \pm 0,43$ & $31 \pm 27,25$ & $4,64 \pm 3,53$ \\
\hline \multirow{3}{*}{ Kodjoboué } & SS & $30,35 \pm 1,50$ & $21,94 \pm 1,24$ & $6,50 \pm 0,50$ & $117,50 \pm 11,83$ & $6,45 \pm 3,39$ \\
\hline & SP & $27,68 \pm 1,85$ & $24,48 \pm 4,32$ & $6,52 \pm 0,71$ & $91,50 \pm 35,90$ & $6,17 \pm 1,33$ \\
\hline & Ensemble & $29,20 \pm 1,80$ & $22,10 \pm 3,64$ & $6,5 \pm 0,63$ & $100,5 \pm 28,26$ & $6,31 \pm 2,58$ \\
\hline
\end{tabular}

\section{Composition spécifique}

Au total, 67 espèces de poissons ont été identifiées au cours des huit campagnes d'échantillonnage (Tableau 3). Elles sont reparties en 11 ordres et 30 familles. Les ordres les mieux représentés sont les Perciformes, Siluriformes et Osteoglossiformes avec respectivement 27, 12 et 10 espèces. Les familles les plus diversifiées sont les Mormyridae et les Cichlidae avec 8 espèces chacune. Elles sont suivies des Clariidae (6) et Cyprinidae (5). La présence de toutes ces 67 espèces a été signalée dans le cours principal du fleuve Comoé. Parmi elles, seulement 17 ont été observées dans la lagune Kodjoboué.

\section{Richesse raréfiée}

Dans l'ensemble, la valeur de la richesse raréfiée oscille entre 11,23 (Kodjoboué en saison sèche) et 34,78 (Adjékro en saison sèche). Les plus grandes valeurs en saison sèche ont été enregistrées à Adjékro $(34,78)$, Moosoou $(23,53)$ et Motobé $(21,713)$ tandis les plus grandes valeurs en saison pluvieuse sont observées à Adjekro (29,19), Yaou $(27,71)$, Palm-CI $(24,31)$ et Moossou $(19,94)$. Quelle que soit la saison, Adjékro enregistre les valeurs les plus élevées. Le test de Kruskal-Wallis note qu'il n'y a pas différence significative entre les saisons ( $\mathrm{p}>0,05)$ (Figure 2). 


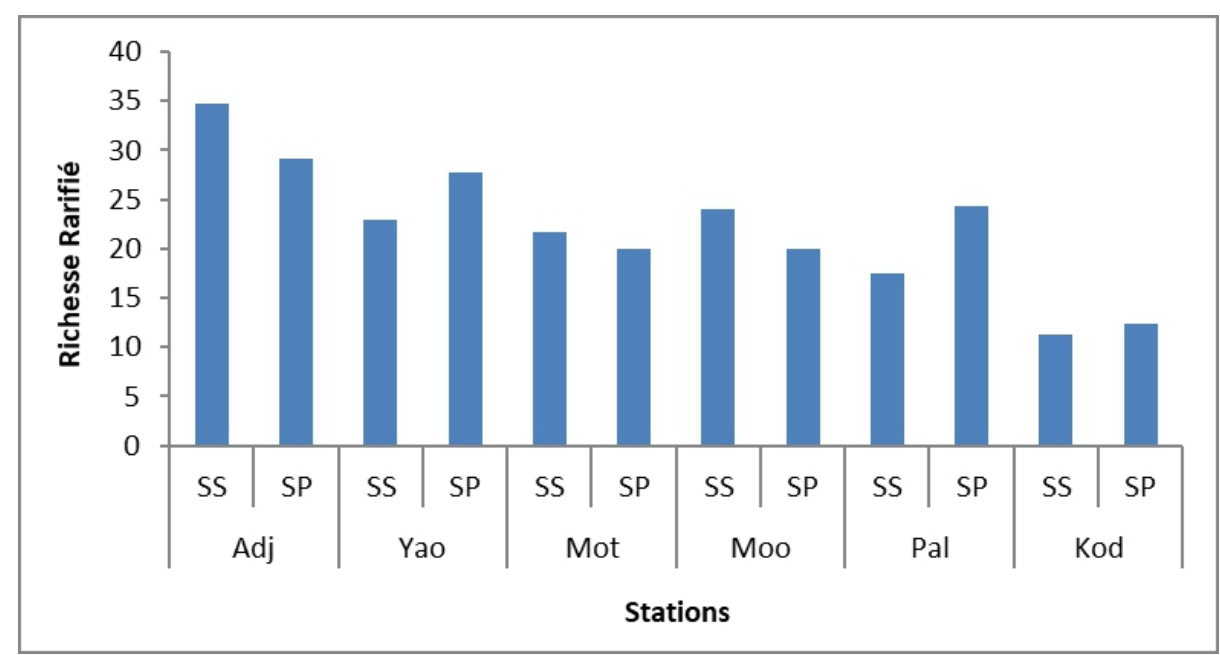

Figure 2 : Variations spatio-temporelle de l'indice raréfié : $\mathbf{S S}=$ saison sèche, $\mathbf{S P}=$ saison pluvieuse

Tableau 3 : Liste des espèces de poissons signalées aux différentes stations échantillonnées dans le bassin inférieur du fleuve Comoé : Adjekro (Adj), Yaou (Yao), Motobé (Mot), Moossou (Moo), Palm-CI (Pal) et Kodjoboué $(\mathbf{K o d}),{ }^{*}=$ présence, $\mathbf{x}=$ signalée pour la première fois dans le bassin du fleuve Comoé

\begin{tabular}{|c|c|c|c|c|c|c|c|c|}
\hline \multirow{2}{*}{ Ordres } & \multirow{2}{*}{ Familles } & \multirow{2}{*}{ Espèces } & \multicolumn{6}{|c|}{ Stations } \\
\hline & & & Adj & Yao & Mot & Moo & Pal & Kod \\
\hline Lepidosireniformes & Protopteridae & Protopterus annectens (Owen, 1839) & $*$ & & & & & \\
\hline \multirow{7}{*}{ Osteoglossiformes } & Notopteridae & Papyrocranus afer (Günther, 1868) & $*$ & $*$ & $*$ & $*$ & $*$ & $*$ \\
\hline & Arapaimidae & Heterotis niloticus (Cuvier, 1829) $^{\mathbf{x}}$ & $*$ & & $*$ & & & \\
\hline & & Brienomyrus brachyistius $(\mathrm{Gill}, 1862)^{\mathbf{x}}$ & & & & & $*$ & \\
\hline & & Marcusenius furcidens (Pellegrin, 1920) & $*$ & & & & & \\
\hline & Mormyridae & Marcusenius senegalensis (Steindachner, 1870) & $*$ & $*$ & $*$ & $*$ & $*$ & $*$ \\
\hline & & Marcusenius ussheri (Günther, 1867) & $*$ & $*$ & $*$ & $*$ & $*$ & $*$ \\
\hline & & Mormyrops anguilloides (Linnaeus, 1758) & $*$ & $*$ & $*$ & & $*$ & \\
\hline
\end{tabular}




\begin{tabular}{|c|c|c|c|c|c|c|c|c|}
\hline & & Mormyrus rume Valenciennes, 1847 & $*$ & & & $*$ & & \\
\hline & & Petrocephalus bovei (Valenciennes, 1847) & $*$ & & $*$ & $*$ & $*$ & \\
\hline & & Pollimyrus isidori (Valenciennes, 1847) & $*$ & $*$ & $*$ & $*$ & $*$ & $*$ \\
\hline Clupeiformes & Clupeidae & Pellonula leonensis Boulenger, 1916 & $*$ & $*$ & $*$ & $*$ & $*$ & \\
\hline Elopiformes & Elopidae & Elops lacerta Valenciennes, 1946 & $*$ & $*$ & $*$ & $*$ & $*$ & \\
\hline \multirow{6}{*}{ Characiformes } & Hepsetidae & Hepsetus odoe (Bloch, 1794) & $*$ & $*$ & $*$ & $*$ & $*$ & $*$ \\
\hline & & Brycinus imberi (Peters, 1852) & & & & * & & \\
\hline & & Brycinus macrolepidotus Valenciennes, 1850 & $*$ & $*$ & $*$ & $*$ & $*$ & \\
\hline & & Brycinus nurse (Rüppell, 1832) & $*$ & $*$ & $*$ & & $*$ & \\
\hline & & Brycinus longipinnis (Günther, 1864) & $*$ & $*$ & $*$ & $*$ & $*$ & $*$ \\
\hline & Distichodontidae & Distichodus rostratus Günther, 1864 & $*$ & $*$ & $*$ & $*$ & $*$ & \\
\hline \multirow{5}{*}{ Cypriniformes } & \multirow{5}{*}{ Cyprinidae } & Clypeobarbus hypsolepis (Daget, 1959) & & & $*$ & & & \\
\hline & & Enteromius ablabes (Bleeker, 1863) & & $*$ & $*$ & & & $*$ \\
\hline & & Enteromius macrops (Boulenger, 1911) & $*$ & $*$ & $*$ & * & $*$ & * \\
\hline & & Labeo coubie Rüppell, 1832 & $*$ & $*$ & $*$ & $*$ & * & \\
\hline & & Labeo senegalensis Valenciennes, 1842 & $*$ & & $*$ & & $*$ & \\
\hline \multirow{5}{*}{ Siluriformes } & \multirow{2}{*}{ Claroteidae } & Chrysichthys maurus (Valenciennes, 1840) & $*$ & $*$ & $*$ & & $*$ & \\
\hline & & Chrysichthys nigrodigitatus (Lacépède, 1803) & $*$ & $*$ & $*$ & $*$ & $*$ & $*$ \\
\hline & \multirow{3}{*}{ Schilbeidae } & Parailia pellucida (Boulenger, 1901) & $*$ & $*$ & $*$ & $*$ & $*$ & $*$ \\
\hline & & Schilbe intermedius Rüppell, 1832 & $*$ & $*$ & $*$ & $*$ & $*$ & \\
\hline & & Schilbe mandibularis (Günther, 1867) & $*$ & $*$ & $*$ & $*$ & $*$ & \\
\hline
\end{tabular}

Tableau 3 (suite) :

\begin{tabular}{|c|c|c|c|c|c|c|c|c|}
\hline \multirow{2}{*}{ Ordres } & \multirow{2}{*}{ Familles } & \multirow{2}{*}{ Espèces } & \multicolumn{6}{|c|}{ Stations } \\
\hline & & & Adj & Yao & Mot & Moo & Pal & Kod \\
\hline \multirow{6}{*}{ Siluriformes } & \multirow{6}{*}{ Clariidae } & Clarias anguillaris (Linnaeus, 1758) & $*$ & $*$ & $*$ & $*$ & $*$ & \\
\hline & & Clarias buettikoferi Steindachner, 1894 & $*$ & & $*$ & $*$ & & \\
\hline & & Clarias ebriensis Pellegrin, 1920 & & $*$ & & & & \\
\hline & & Clarias gariepinus (Burchell, 1822) & $*$ & & & & & \\
\hline & & Gymnallabes $\mathrm{sp}^{\mathrm{x}}$. & & & & & & \\
\hline & & Heterobranchus isopterus Bleeker, 1863 & $*$ & & & & & \\
\hline
\end{tabular}




\begin{tabular}{|c|c|c|c|c|c|c|c|c|}
\hline & Mochokidae & Synodontis bastiani Daget, 1948 & $*$ & & & & & \\
\hline \multirow{2}{*}{ Mugiliformes } & \multirow{2}{*}{ Mugilidae } & Neochelon falcipinnis (Valenciennes, 1836) & & $*$ & $*$ & $*$ & $*$ & \\
\hline & & Mugil cephalus Linnaeus, $1758^{\mathbf{x}}$ & & $*$ & & & & \\
\hline Syngnathiformes & Syngnathidae & Microphis brachyurus (Bleeker, 1854) ${ }^{\mathbf{x}}$ & & & $*$ & & & \\
\hline Pleuronectiformes & Cynoglossidae & Cynoglossus senegalensis (Kaup, 1858) & $*$ & $*$ & & & & \\
\hline \multirow{21}{*}{ Perciformes } & Channidae & Parachanna obscura (Günther, 1861) & $*$ & & & $*$ & & \\
\hline & \multirow{4}{*}{ Eleotridae } & Eleotris senegalensis Steindachner, 1870 & $*$ & $*$ & $*$ & $*$ & \multirow{4}{*}{$*$} & \\
\hline & & Eleotris vittata Duméril, 1861 & $*$ & $*$ & & & & \\
\hline & & Kribia kribensis (Boulenger, 1907) & $*$ & $*$ & $*$ & $*$ & & \\
\hline & & Dormitator lebrotonis (Steindachner, 1870) ${ }^{\mathbf{x}}$ & & & $*$ & & & \\
\hline & Gobiidae & Awaous lateristriga (Duméril, 1861) & & & & $*$ & & \\
\hline & \multirow{9}{*}{ Cichlidae } & Coptodon guineensis (Günther, 1862) & $*$ & $*$ & & & & $*$ \\
\hline & & Coptodon guineensis x Coptodon zillii & $*$ & & & & $*$ & $*$ \\
\hline & & Hemichromis bimaculatus Gill, 1862 & $*$ & $*$ & $*$ & & & \\
\hline & & Hemichromis fasciatus Peters, 1857 & $*$ & $*$ & $*$ & $*$ & $*$ & $*$ \\
\hline & & Oreochromis niloticus (Linnaeus, 1758) & $*$ & & & & & \\
\hline & & Pelmatolapia mariae (Boulenger, 1899) & $*$ & $*$ & $*$ & & & $*$ \\
\hline & & Sarotherodon melanotheron Rüppell, 1852 & $*$ & $*$ & & & \multirow{3}{*}{$*$} & $*$ \\
\hline & & Tylochromis intermedius (Boulenger, 1916) & $*$ & & & & & $*$ \\
\hline & & Tylochromis jentinki (Steindachner, 1895) & $*$ & & & $*$ & & $*$ \\
\hline & Anabantidae & Ctenopoma petherici Günther, 1864 & $*$ & $*$ & $*$ & $*$ & $*$ & \\
\hline & Haemulidae & Pomadasys jubelini (Cuvier, 1830) ${ }^{\mathbf{x}}$ & $*$ & $*$ & & & & \\
\hline & Monodactylidae & Monodactylus sebae (Cuvier, 1829) & $*$ & & & $*$ & $*$ & \\
\hline & Polynemidae & Polydactylus quadrifilis (Cuvier 1829) & $*$ & $*$ & $*$ & $*$ & & \\
\hline & \multirow{2}{*}{ Sciaenidae } & Pseudotolithus elongatus (Bowdich, 1825) & $*$ & $*$ & & & & \\
\hline & & Pseudotolithus senegalensis (Valenciennes, 1833) & & $*$ & $*$ & & & \\
\hline
\end{tabular}

Tableau 3 (suite) :

\begin{tabular}{|c|c|c|c|c|c|c|c|c|}
\hline \multirow{2}{*}{ Ordres } & \multirow{2}{*}{ Familles } & \multirow{2}{*}{ Espèces } & \multicolumn{6}{|c|}{ Stations } \\
\hline & & & $\overline{\text { Adj }}$ & Yao & Mot & Moo & Pal & Kod \\
\hline Perciformes & Carangidae & Caranx rhonchus (Geoffroy Saint-Hilaire, 1817$)^{\mathbf{x}}$ & & * & & & & \\
\hline
\end{tabular}


Trachinotus teraia Cuvier, $1832^{\mathrm{x}}$

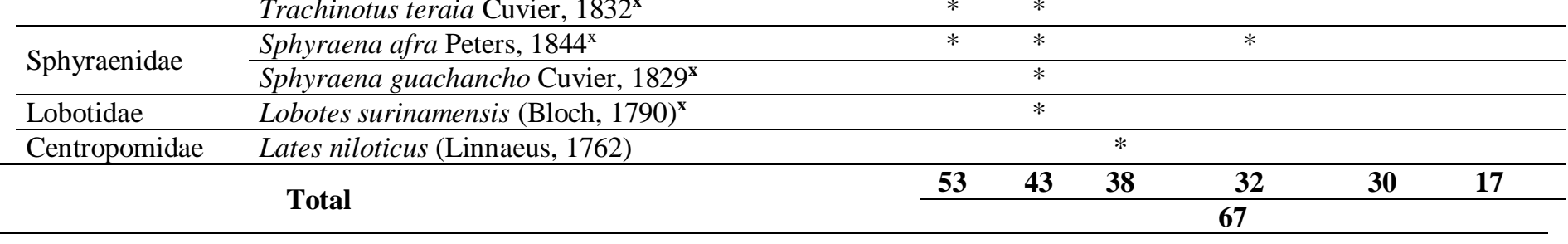

\section{Analyse de la diversité du peuplement}

Les valeurs extrêmes de l'indice de Shannon $(0,05$ et 1,38) ont été enregistrées à la station d'Adjékro. Ces valeurs obtenues indiquent que le peuplement est peu diversifié. Relativement à l'équitabilité, les plus faibles valeurs se trouvent à Adjékro, Kodjoboué $(0,07)$ et Yaou $(0,24)$. Ces valeurs montrent que la distribution du peuplement est régulière. Le test de Kruskal-Wallis indique qu'il n'y a pas de différence significative entre les stations en ce qui concerne l'indice de Shannon ( $p=0,75>0,05)$ et de l'équitabilité $(p=0,94>0,05)$ (Figure 3).
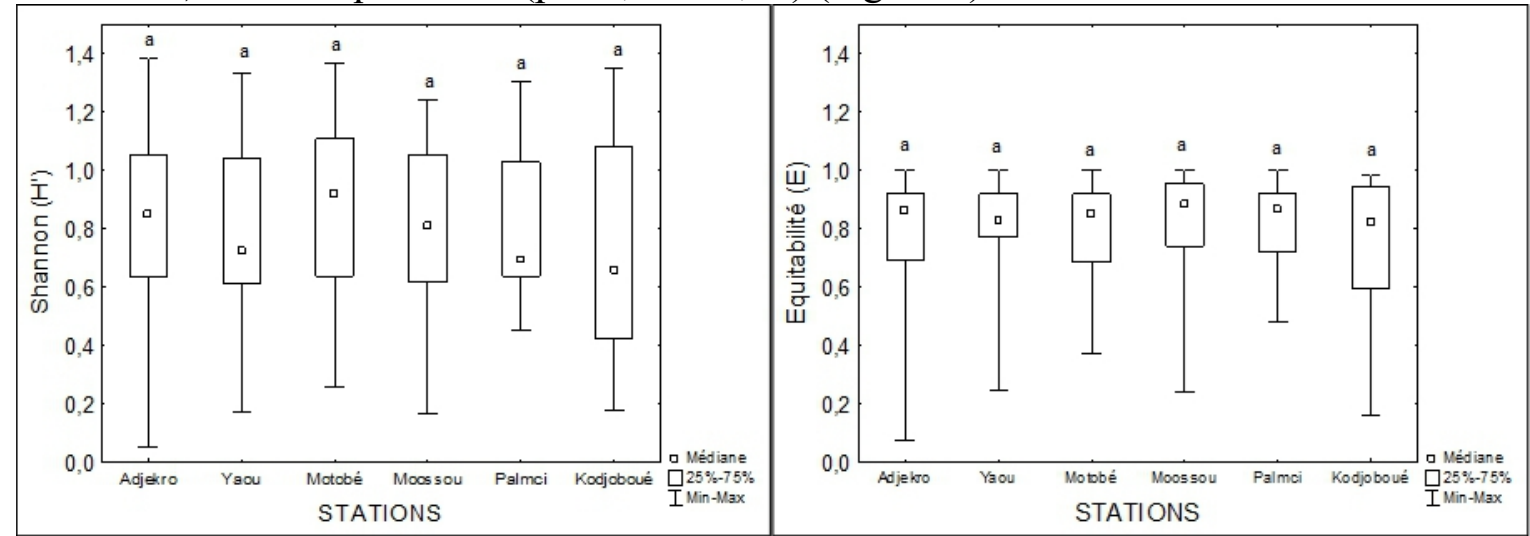

Figure 3 : Variations spatiales des indices de Shannon et d'équitabilité du peuplement ichtyologique aux différentes stations. 
Aux stations d'Adjékro, Motobé, Moossou, les valeurs maximales de l'indice de Shannon et de l'équitabilité ont été observées en saison pluvieuse. En revanche aux stations de Yaou, Palm-CI et Kodjoboué les valeurs maximales de ces indices ont été enregistrées en saison sèche. Le test U de Mann-Whitney révèle qu'il n'existe aucune différence significative entre les valeurs de ces indices pour chacune des stations d'une saison à l'autre (Figure 4).

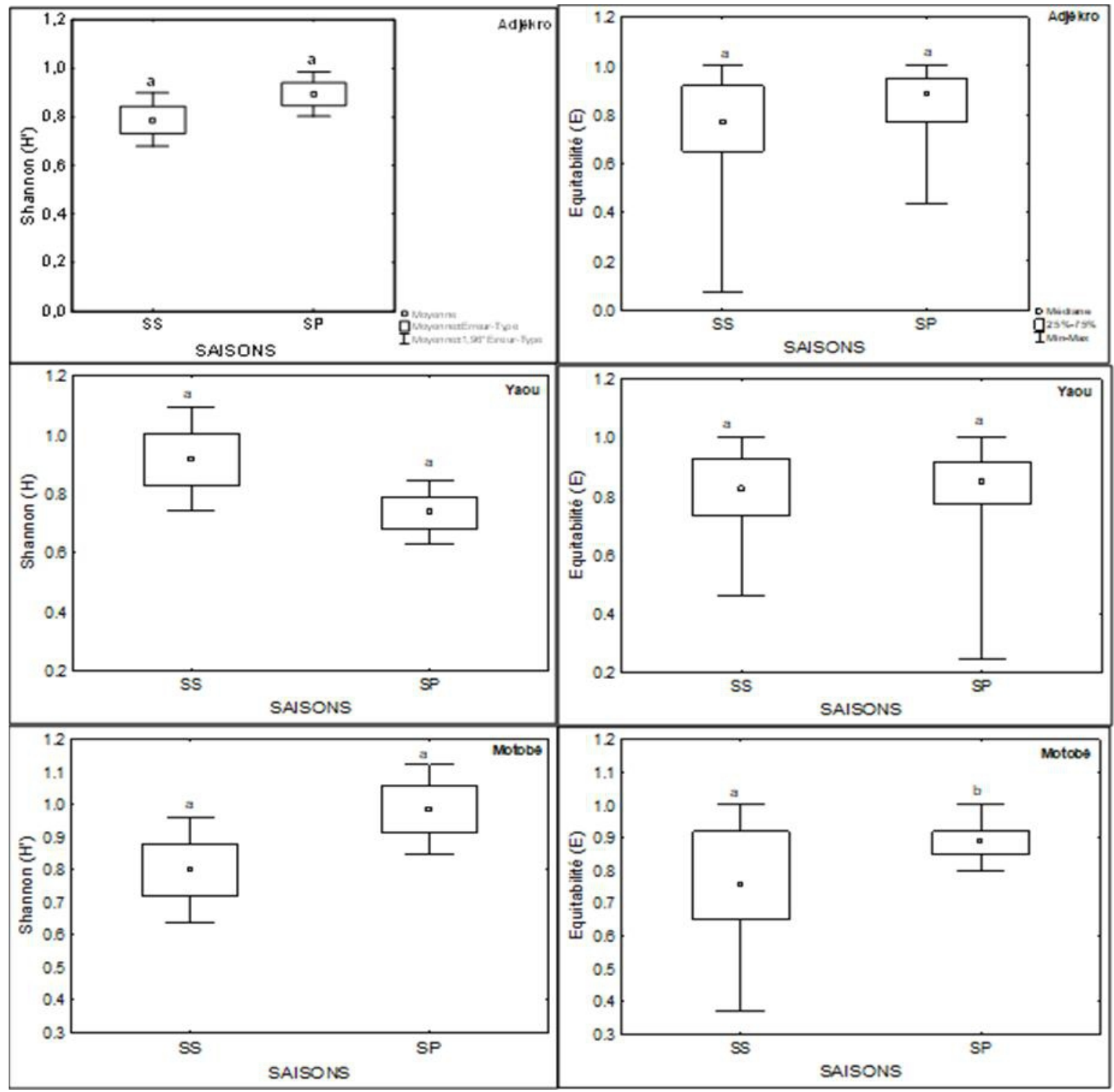

Figure 4: Variations saisonnières des indices de Shannon et d'équitabilité du peuplement ichtyologique aux différentes stations : $\mathbf{S S}$ = saison sèche, $\mathbf{S P}=$ saison pluvieuse 

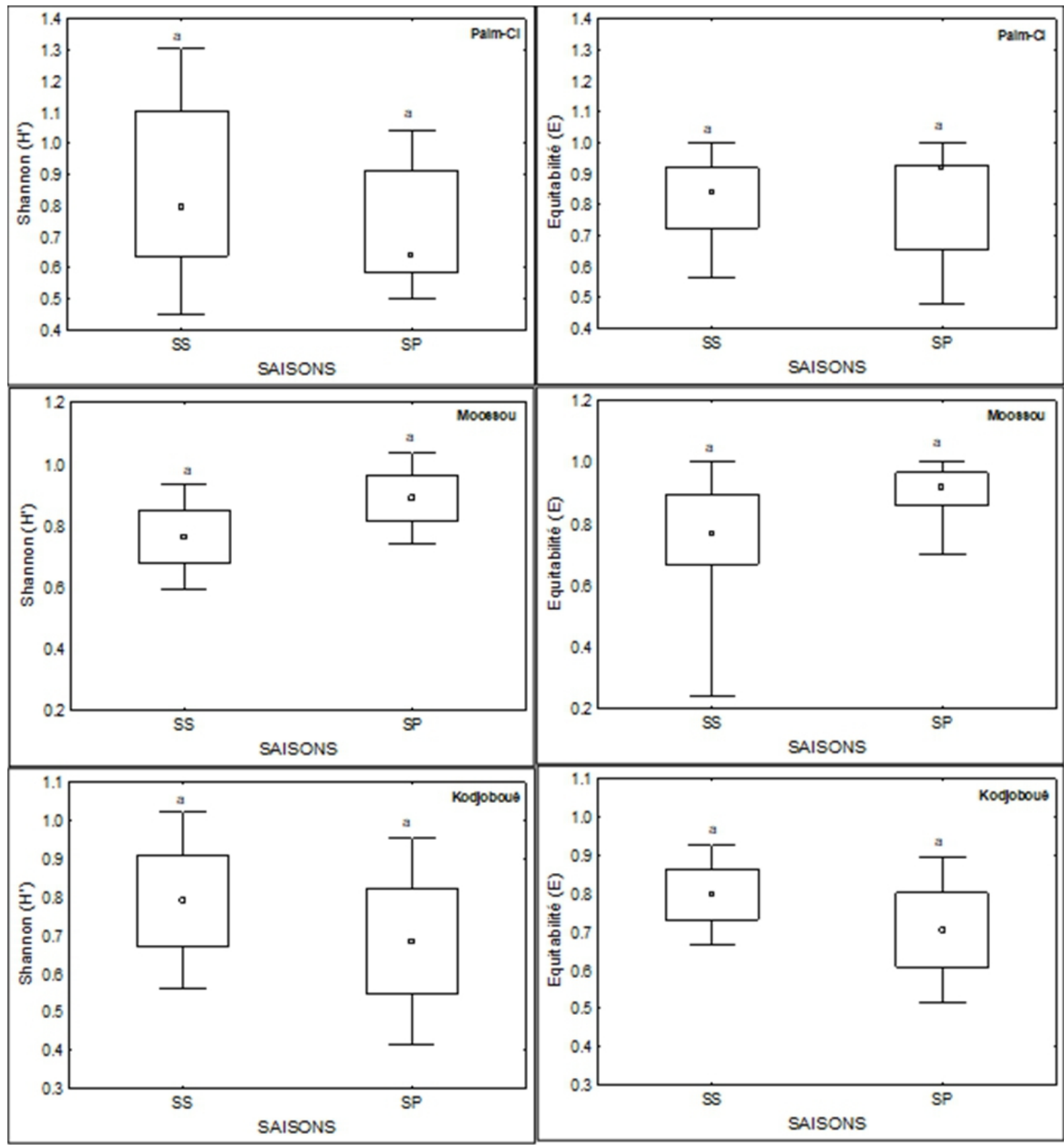

Figure 4 (suite) :

Les proportions de catégories d'espèces de poissons par station sont présentées dans le tableau 4. A Adjékro plus de la moitié $(52,83 \%)$ des espèces pêchées sont fréquentes dans les captures. Par contre, à Motobé, Moossou et Palm-CI, plus de $40 \%$ des poissons sont accidentellement pêchés (40 à 47,37\%). A ces sites, les espèces constantes représentent entre 29 et 37 
$\%$ de la richesse ichtyofaunique. A Yaou, les espèces accidentelles (39,54 \% et accessoires $(34,88 \%)$ dominent la population. A Kodjoboué les espèces accessoires $(47,06 \%)$ et constantes $(41,18 \%)$ sont majoritaires.

Tableau 4 : Proportions (\%) des catégories d'espèces définies sur la base de l'occurrence dans les sites échantillonnés

\begin{tabular}{lcccccc}
\hline Catégories & Adjékro & Yaou & Motobé & Moossou & Palm-CI & Kodjoboué \\
\hline Constantes & 52,83 & 25,58 & 28,95 & 34,38 & 36,67 & 41,18 \\
Accessoires & 22,64 & 34,88 & 23,68 & 21,87 & 23,33 & 47,06 \\
Accidentelles & 24,53 & 39,54 & 47,37 & 43,75 & 40 & 11,76 \\
\hline
\end{tabular}

En termes d'abondance, sur l'ensemble des stations échantillonnées, un total de 7265 spécimens de poissons a été capturé. La plus forte abondance (2428 individus) a été enregistrée à la station d'Adjékro et la plus faible (583 individus) à la station de Moossou (Tableau 5).

$\mathrm{Au}$ niveau saisonnier, les plus fortes abondances ont été obtenues en saison sèche aux stations d'Adjékro, Motobé, Moossou et Palm-CI. A Yaou et Kodjoboué le contraire est observé.

Tableau 5 : Récapitulatif des abondances saisonnières et spatiales des poissons capturés aux différentes stations

\begin{tabular}{lccc}
\hline \multirow{2}{*}{ Stations } & \multicolumn{2}{c}{ Abondances par station et par saison } & \\
\cline { 2 - 3 } & Saison sèche & Saison pluvieuse & Abondance par station \\
\hline Adjékro & 1100 & 1328 & 2428 \\
Yaou & 304 & 881 & 1185 \\
Motobé & 732 & 375 & 1107 \\
Moossou & 344 & 239 & 583 \\
Palm-CI & 464 & 261 & 725 \\
Kodjoboué & 591 & 646 & 1237 \\
\hline
\end{tabular}

Les différentes valeurs de l'indice de Jaccard calculées entre les sites échantillonnés sont consignées dans le tableau 6. Elles varient entre 0,28 et 0,62 . On observe une similarité entre certaines stations situées sur le cours principal du fleuve prises deux à deux tandis qu'aucune similarité n'est observée entre la station de Kodjoboué et les autres. Les similarités ont été observées entre les couples de stations Motobé-Palm-CI $(0,62)$, MoossouPalm-CI $(0,59)$, Adjékro-Yaou (0,57), Yaou-Motobé $(0,56)$, MotobéMoossou $(0,52)$ et Adjékro-Palm-CI $(0,51)$.

La régression multiple pas à pas ascendante a été utilisée pour mettre en relation les abondances des espèces de poissons et les caractéristiques de l'environnement. Les résultats de cette analyse sont présentés par le tableau 7. La conductivité est positivement corrélée à 27 espèces, la transparence à 13 espèces, le $\mathrm{pH}$ à 8 espèces, l'oxygène dissous à 6 espèces. Par ailleurs, les espèces Enteromius ablabes, Enteromius macrops, Brycinus longipinnis, Tylochromis intermedius et Tylochromis jentinki sont corrélées au $\mathrm{pH}$; 
Clarias anguilaris, Eleotris vittata, Heterotis niloticus, Neochelon falcipinnis, Coptodon guineensis sont négativement corrélées à la transparence. Quant à la température et la conductivité, elles sont respectivement corrélées négativement à Mormyrus rume, parachanna obscura et Parailia pellucida.

Tableau 6 : Comparaison des valeurs de l'indice de similarité de Jaccard calculées entre les différents milieux d'échantillonnage du bassin inférieur du fleuve Comoé

\begin{tabular}{lcccccc}
\hline \multicolumn{1}{c}{ Stations } & Adjékro & Yaou & Motobé & Moossou & Palm-CI & Kodjoboué \\
\hline Adjékro & 1 & & & & & \\
Yaou & 0,57 & 1 & & & & \\
Motobé & 0,49 & 0,56 & 1 & & & \\
Moossou & 0,49 & 0,47 & 0,52 & 1 & & \\
Palm-CI & 0,51 & 0,49 & 0,62 & 0,59 & 1 & \\
Kodjoboué & 0,30 & 0,30 & 0,28 & 0,29 & 0,30 & 1 \\
\hline
\end{tabular}

Tableau 7 : Régression multiple pas à pas mettant en relation les paramètres physiques, chimiques et des taxons dans le bassin inférieur du fleuve Comoé : $\mathrm{R}^{2}=$ coefficient de détermination, $\mathrm{F}=$ coefficient d'inclusion, $\mathrm{t}=$ coefficient de régression, $\mathrm{p}=$ probabilité

\begin{tabular}{|c|c|c|c|c|c|}
\hline Taxons & Paramètres & $\mathbf{t}$ & $\mathbf{R}^{2}$ & $\mathbf{F}$ & $\mathbf{P}$ \\
\hline \multirow[t]{2}{*}{ Enteromius ablabes } & $\mathrm{pH}$ & $-3,57$ & 0,61 & $\mathrm{~F}(3,8)=4,25$ & 0,007 \\
\hline & Conductivité & 3,07 & 0,61 & $\mathrm{~F}(3,8)=4,25$ & 0,015 \\
\hline Enteromius macrops & $\mathrm{pH}$ & $-3,72$ & 0,66 & $\mathrm{~F}(3,8)=5,30$ & 0,005 \\
\hline \multirow[t]{2}{*}{ Brycinus longipinnis } & $\mathrm{pH}$ & $-4,12$ & 0,82 & $\mathrm{~F}(5,6)=5,65$ & 0,006 \\
\hline & Conductivité & 5,23 & 0,93 & $\mathrm{~F}(3,8)=41,73$ & 0,000 \\
\hline Brycinus macrolepidotus & Transparence & 4,20 & 0,93 & $\mathrm{~F}(3,8)=41,73$ & 0,002 \\
\hline Chrysichthys maurus & $\mathrm{O}_{2}$ & 2,91 & 0,60 & $\mathrm{~F}(2,9)=6,84$ & 0,017 \\
\hline Chrysichthys nigrodigitatus & Conductivité & 10,75 & 0,96 & $\mathrm{~F}(4,7)=47,47$ & 0,000 \\
\hline \multirow[t]{2}{*}{ Clarias anguilaris } & Conductivité & 7,71 & 0,88 & $\mathrm{~F}(3,8)=19,85$ & 0,000 \\
\hline & Transparence & $-3,36$ & 0,88 & $\mathrm{~F}(3,8)=19,85$ & 0,009 \\
\hline Clarias gariepinus & Conductivité & 5,73 & 0,86 & $\mathrm{~F}(4,7)=11,37$ & 0,000 \\
\hline \multirow[t]{2}{*}{ Ctenopoma petherici } & Conductivité & 4,21 & 0,74 & $\mathrm{~F}(3,8)=7,66$ & 0,002 \\
\hline & Conductivité & 4,91 & 0,95 & $\mathrm{~F}(4,7)=38,54$ & 0,001 \\
\hline \multirow{2}{*}{ Distichodus rostratus } & Transparence & 3,31 & 0,95 & $\mathrm{~F}(4,7)=38,54$ & 0,010 \\
\hline & $\mathrm{pH}$ & 3,29 & 0,95 & $\mathrm{~F}(4,7)=38,54$ & 0,010 \\
\hline \multirow[t]{2}{*}{ Eleotris vittata } & Conductivité & 6,04 & 0,82 & $\mathrm{~F}(3,8)=12,60$ & 0,000 \\
\hline & Transparence & $-3,27$ & 0,82 & $\mathrm{~F}(3,8)=12,60$ & 0,010 \\
\hline Elops lacerta & Conductivité & 3,30 & 0,72 & $\mathrm{~F}(4,7)=4,69$ & 0,013 \\
\hline Hemichromis bimaculatus & Conductivité & 3,87 & 0,77 & $\mathrm{~F}(4,7)=5,99$ & 0,006 \\
\hline Hemichromis fasciatus & Conductivité & 3,65 & 0,60 & $\mathrm{~F}(2,9)=6,91$ & 0,005 \\
\hline Hepsetus odoe & Transparence & 2,49 & 0,47 & $\mathrm{~F}(2,8)=3,65$ & 0,037 \\
\hline \multirow{2}{*}{ Heterotis niloticus } & Conductivité & 8,63 & 0,90 & $\mathrm{~F}(3,8)=25,41$ & 0,000 \\
\hline & Transparence & $-3,05$ & 0,90 & $\mathrm{~F}(3,8)=25,41$ & 0,015 \\
\hline Kribia kribensis & Conductivité & 6,49 & 0,91 & $\mathrm{~F}(4,7)=18,48$ & 0,000 \\
\hline \multirow[t]{2}{*}{ Labeo coubie } & Conductivité & 10,04 & 0,98 & $\mathrm{~F}(4,7)=88,30$ & 0,000 \\
\hline & Transparence & 4,79 & 0,98 & $\mathrm{~F}(4,7)=88,30$ & 0,001 \\
\hline Labeo senegalensis & Température & 3,53 & 0,82 & $\mathrm{~F}(6,5)=3,72$ & 0,010 \\
\hline Neochelon falcipinnis & Transparence & $-3,29$ & 0,82 & $\mathrm{~F}(4,7)=7,87$ & 0,010 \\
\hline Marcusenius furcidens & Conductivité & 5,67 & 0,86 & $\mathrm{~F}(4,7)=11,13$ & 0,000 \\
\hline
\end{tabular}


Tableau 7: (suite)

\begin{tabular}{|c|c|c|c|c|c|}
\hline Taxons & Paramètres & $\mathbf{t}$ & $\mathbf{R}^{2}$ & $\mathbf{F}$ & $\mathbf{P}$ \\
\hline Marcusenius senegalensis & Conductivité & 2,88 & 0,73 & $\mathrm{~F}(3,8)=7,20$ & 0,020 \\
\hline \multirow{3}{*}{ Marcusenius ussheri } & Conductivité & 11,75 & 0,97 & $\mathrm{~F}(4,7)=74,80$ & 0,000 \\
\hline & Température & 2,45 & 0,97 & $\mathrm{~F}(4,7)=74,80$ & 0,040 \\
\hline & $\mathrm{O}_{2}$ & 3,65 & 0,97 & $\mathrm{~F}(4,7)=74,80$ & 0,008 \\
\hline Monodactylus sebae & Conductivité & 6,58 & 0,84 & $\mathrm{~F}(2,9)=24,63$ & 0,000 \\
\hline \multirow{3}{*}{ Mormyrops anguilloides } & Transparence & 3,80 & 0,96 & $\mathrm{~F}(5,6)=30,97$ & 0,008 \\
\hline & Conductivité & 3,87 & 0,96 & $\mathrm{~F}(5,6)=30,97$ & 0,008 \\
\hline & Température & 2,56 & 0,96 & $\mathrm{~F}(5,6)=30,97$ & 0,040 \\
\hline Mormyrus rume & Température & $-2,83$ & 0,87 & $F(6,5)=5,89$ & 0,030 \\
\hline \multirow[t]{2}{*}{ Papyrocranus afer } & Conductivité & 8,97 & 0,96 & $\mathrm{~F}(3,8)=67,96$ & 0,000 \\
\hline & Transparence & 2,54 & 0,96 & $\mathrm{~F}(3,8)=67,96$ & 0,035 \\
\hline \multirow[t]{2}{*}{ Parachanna obscura } & Conductivité & 12,71 & 0,98 & $\mathrm{~F}(6,5)=54,20$ & 0,000 \\
\hline & Température & $-2,89$ & 0,98 & $\mathrm{~F}(6,5)=54,20$ & 0,034 \\
\hline \multirow[t]{2}{*}{ Parailia pellucida } & $\mathrm{pH}$ & 5,21 & 0,82 & $\mathrm{~F}(4,7)=8,08$ & 0,007 \\
\hline & Conductivité & $-2,44$ & 0,82 & $\mathrm{~F}(4,7)=8,08$ & 0,044 \\
\hline Pellonula leonensis & $\mathrm{pH}$ & 2,33 & 0,53 & $\mathrm{~F}(3,8)=3,08$ & 0,047 \\
\hline Polydactylus quadrifilis & Conductivité & 3,54 & 0,78 & $\mathrm{~F}(3,8)=9,69$ & 0,007 \\
\hline Protopterus annectens & Conductivité & 11,04 & 0,98 & $\mathrm{~F}(4,7)=84,74$ & 0,000 \\
\hline \multirow[t]{2}{*}{ Pseudotolithus elongatus } & Transparence & 3,21 & 0,79 & $\mathrm{~F}(3,8)=10,53$ & 0,012 \\
\hline & $\mathrm{O}_{2}$ & 2,48 & 0,79 & $\mathrm{~F}(3,8)=10,53$ & 0,038 \\
\hline \multirow[t]{2}{*}{ Sarotherodon melanotheron } & $\mathrm{O}_{2}$ & 2,49 & 0,79 & $\mathrm{~F}(4,7)=6,91$ & 0,041 \\
\hline & $\mathrm{O}_{2}$ & 2,82 & 0,72 & $\mathrm{~F}(4,7)=4,47$ & 0,026 \\
\hline \multirow[t]{2}{*}{ Coptodon guineensis } & Transparence & $-2,46$ & 0,72 & $\mathrm{~F}(4,7)=4,47$ & 0,043 \\
\hline & Conductivité & 2,60 & 0,72 & $\mathrm{~F}(4,7)=4,47$ & 0,035 \\
\hline \multirow[t]{2}{*}{ C. guineensis $x$ C. zillii } & Conductivité & 4,37 & 0,93 & $\mathrm{~F}(4,7)=24,46$ & 0,003 \\
\hline & Transparence & 2,70 & 0,93 & $\mathrm{~F}(4,7)=24,46$ & 0,031 \\
\hline Pelmatolapia mariae & Conductivité & 22,15 & 0,99 & $\mathrm{~F}(5,6)=223,36$ & 0,000 \\
\hline Trachinotus teraia & $\mathrm{O}_{2}$ & 2,58 & 0,53 & $\mathrm{~F}(2,9)=5,07$ & 0,029 \\
\hline Tylochromis intermedius & $\mathrm{pH}$ & $-4,0$ & 0,68 & $\mathrm{~F}(9,8)=5,82$ & 0,003 \\
\hline Tylochromis jentinki & $\mathrm{pH}$ & $-3,47$ & 0,69 & $\mathrm{~F}(4,7)=3,92$ & 0,010 \\
\hline
\end{tabular}

\section{Discussion}

Les données de la physico-chimie des eaux sont indispensables à la vie des poissons. Selon Chikou et al. (2008), les deux paramètres les plus importants pour la croissance des poissons sont la température et l'oxygène dissous. Par ailleurs, Jensen (1957) rapporte qu'une diminution de la température, si infime soit-elle, ralentit la croissance chez les poissons. Les données abiotiques du bassin inférieur du fleuve Comoé indiquent que les températures dans les stations prospectées avoisinent celles enregistrées dans d'autres cours d'eau tels que $1^{\prime}$ Agnéby $\left(26,27^{\circ} \mathrm{C}\right)$ et la Bia $\left(26,10^{\circ} \mathrm{C}\right)$ selon Gooré Bi (1998). Globalement ces données concordent avec celles de Lemoalle (1999) qui font observer qu'en Afrique intertropicale les températures moyennes sont élevées et le plus souvent supérieures à $20^{\circ} \mathrm{C}$. Iltis \& Lévêque (1982) ont noté que la température des cours d'eau dans cette zone de la Côte d'Ivoire descend rarement en dessous de $25^{\circ} \mathrm{C}$. Les valeurs 
de température enregistrées à toutes les stations tant dans l'affluent que dans le cours principal indiquent que les eaux sont chaudes. Les milieux étudiés ayant des canopées totalement ouvertes, ils reçoivent de ce fait directement la lumière solaire qui réchauffe les eaux. En effet, Millet (1984), Durand \& Chantraine (1982), Issola et al. (2008) et Konan et al. (2008) font savoir que les rayons solaires ont la capacité de traverser l'épaisseur d'eau pour la réchauffer de façon homogène.

Dans l'ensemble, les valeurs de température, $\mathrm{pH}$, oxygène dissous et transparence sont similaires à celles obtenues par Keumean et al. (2013) dans l'eau de l'estuaire du même fleuve. Ces auteurs ont noté, comme c'est le cas dans la présente étude, les mêmes évolutions spatiales des valeurs de ces paramètres de l'amont vers l'aval. Les valeurs de transparence indiquent que l'eau est plus transparente dans l'affluent Kodjoboué que dans le cours principal. Cette situation serait due au fait que le cours du lit principal reçoit des particules issues de divers horizons. En transportant des particules de terre, les eaux de ruissellement transportent aussi les éléments nutritifs dissous associés à ces sédiments (Somé et al., 2008). Ces résultats corroborent ceux de Gourène et al. (2002) et de Iltis \& Lévêque (1982) qui indiquent que la transparence est en général plus faible en aval des cours d'eau côtiers. A Kodjoboué l'eau donne l'impression d'être stagnante favorisant ainsi la décantation des particules dissoutes.

Les paramètres liés à la minéralisation ( $\mathrm{pH}$ et conductivité) et la transparence apparaissent les plus discriminants dans la répartition des espèces ichtyologiques dans les eaux. Toutes les stations présentent des valeurs de $\mathrm{pH}$ proches de la neutralité. Keumean et al. (2013) ont obtenu des résultats similaires. Les valeurs de conductivité varient entre 19 et $127 \mu \mathrm{S} / \mathrm{cm}$. Cette situation serait due au fait que les rivières drainent en amont un substrat argilolimoneux et les terres agricoles favorisant l'enrichissement des cours d'eau en sels nutritifs (Konan, 2008).

Le taux d'oxygène dissous oscille entre 1,14 et $11,50 \mathrm{mg} / \mathrm{L}$. La même gamme de valeurs et la même évolution spatiale ont été observées par Keumean et al., 2013 dans la même zone du fleuve. Ces valeurs sont inférieures à celles de Sankaré \& Etien (1991) qui sont supérieures à 4 mg/L sur tout l'estuaire. Selon Matthes (1964), les variations de la quantité d'oxygène dissous sont sous l'influence des facteurs tels que le vent, la profondeur du cours d'eau, la vitesse du courant et la présence de dépôts réducteurs. Ainsi, les rejets des complexes agro-industriels, notamment ceux de COPALTECH et des Plantations Jean EGLIN S.A semblent contribuer à la réduction de la quantité d'oxygène dissous dans les eaux environnantes. A cela on pourrait ajouter la présence excessive de végétaux aquatiques envahissants. Des études antérieures dans cette zone géographique par Niamien-Ebrottié (2010), Konan (2008) et Edia (2008) sur les rivières Soumié, Eholié, Ehania 
et Noé, indiquent les mêmes gammes de valeurs. En effet, les résultats des différentes mesures physiques et chimiques ont montré que les valeurs étaient approximativement les mêmes dans les quatre cours d'eau échantillonnés, malgré quelques variations observées d'une station à l'autre et d'une saison à une autre. Cette ressemblance dans les valeurs des paramètres physiques et chimiques peut être recherchée au niveau des conditions naturelles des bassins versants qui sont assez semblables dans cette région. Par ailleurs, Yao (2006) sur Comoé obtient des valeurs presque semblables.

La présente étude a recensé 67 espèces de poissons dont 16 signalées pour la première fois dans tout le bassin du fleuve Comoé. Ce qui suggère la présence d'une importante diversité spécifique de poissons dans le fleuve Comoé. Parmi les 67 espèces mentionnées, une d'entre elles n'a pu être formellement identifiée ; il s'agit de Gymnallabes sp. La présence dans les captures de 16 espèces signalées pour la première fois dans le bassin du fleuve Comoé pourrait s'expliquer par les mouvements migratoires (volontaires, passifs ou subis) d'une retenue d'eau à une autre, d'un cours d'eau à un autre ou dans le même cours d'eau. En réalité, peu de poissons sont sédentaires. Paugy et al. (2003a et 2003b) notent que ces espèces de poissons remontent les cours inférieurs des fleuves, parcourant parfois de longues distances pour leurs besoins biologiques en relation avec l'alimentation et la reproduction. La présence de Heterotis niloticus s'explique par son introduction en Côte d'Ivoire à partir du Cameroun pour l'aquaculture et la pêche artisanale (Moreau et al., 1988). Des travaux antérieurs réalisés par Daget \& Iltis (1965) ; Teugels et al. (1988) ; Paugy et al. (1994) et Yao et al. (2005), il ressort que la richesse spécifique des poissons est de 97 espèces dans l'ensemble du fleuve Comoé. Seuls les travaux de Yao (2006) stratifient la richesse spécifique selon les cours supérieur, moyen et inférieur. Dans cette base, 16 espèces susceptibles d'être présentes dans le cours inférieur du fleuve (Yao, 2006) n'ont pas été rencontrées au cours de la présente étude. Il s'agit de : Amphilius atesuensis Boulenger, 1904 ; Rhexipanchax schioetzi (Scheel, 1968), Epiplatys chaperi (Sauvage, 1882), Epiplatys etzeli Berkenkamp, 1975 ; Fundulopanchax walkeri (Boulenger, 1911), Chromidotilapia guntheri (Sauvage, 1882), Thysochromis ansorgii (Boulenger, 1901); Kribia nana (Boulenger, 1901), Mastacembelus nigromarginatus Boulenger, 1898 ; Hydrocynus forskahlii (Cuvier, 1819), Micralestes comoensis Poll \& Roman, 1967 ; Micralestes elongatus Daget, 1957 ; Nannocharax fasciatus Günther, 1867 ; Enteromius trispilos (Bleeker, 1863), Enteromius walkeri (Boulenger, 1904) ; Labeobarbus wurtzi (Pellegrin, 1908). L'absence des 16 espèces dans la présente étude pourrait se justifier, d'une part, par le fait que ces espèces se soient retirées dans des habitats particuliers n'ayant pas été prospectés au cours de la présente étude (Lalèyè et al., 2004). En effet, selon Lévêque (1995), au cours de sa vie, et en fonction de son activité journalière ou saisonnière, une 
même espèce de poisson peut occuper successivement plusieurs types d'habitats. D'autre part, il pourrait s'agir des avantages et mode d'échantillonnage notamment les engins utilisés et les zones prospectées. Quels que soient les techniques et les engins de capture choisis, il est évident que l'engin de pêche d'échantillonnage idéal n'existe pas (Diouf, 1994). Dans la présente étude un échantillonnage passif au moyen des pêches expérimentales et artisanales dont les engins sont essentiellement les filets maillants, nasses, pièges, palangres a été fait. Par contre, Yao (2006) a combiné pêche passive et pêche électrique dans les zones peu profondes et dans les petits affluents.

Les valeurs de l'indice de Shannon-Weaver (H') et l'équitabilité (E) montrent que les peuplements ichtyologiques sont équilibrés dans l'ensemble. Cela traduit qu'il n'y a pas de taxon largement dominant. La comparaison de la richesse raréfiée entre les sites d'échantillonnage permet de déduire que la station d'Adjékro apparaît comme la plus diversifiée et que tous les sites sont stables et mieux organisés. Ce constat serait dû à une meilleure répartition des taxons dans les sites. Ces résultats pourraient s'expliquer par le fait dans la partie sud de la Côte d'Ivoire, il n'y a réellement pas absence de pluies quelles que soient les saisons.

L'analyse des proportions (\%) des catégories définies sur la base de l'occurrence montre qu'en général les espèces accessoires et accidentelles sont les plus représentées dans les stations d'échantillonnage sauf à Adjékro. Cela pourrait s'expliquer par le fait que c'est seulement à ce campement que des pêcheurs débarquent régulièrement.

Les données de terrain font observer une abondance numérique des espèces naturellement de petite taille qui échappent aux filets maillants des pêcheurs tels que Brycinus longipinnis, Pollimyrus isidori, Parailia pellucida, Pellonula leonensis et de juvéniles d'espèces naturellement de grande taille tel que Chrysichthys nigrodigitatus, Chrysichthys maurus, Distichodus rostratus, Schilbe intermedius, Schilbe mandibularis, etc. Cette situation serait le fait d'une perturbation induite par la pression de la pêche, les activités anthropiques, la mortalité naturelle due à la prédation, au vieillissement et à la maladie (Pauly, 1980 ; Caillart \& Morize, 1991).

L'analyse comparative des milieux, réalisée à partir de l'indice de similarité de Jaccard montre que les plus grandes similarités ont été observées pour les couples de stations situées sur le cours principal du fleuve. Cette similarité s'expliquerait par une homogénéité spatiale et saisonnière des conditions de l'environnement. En revanche, il n'y a aucune similarité entre la station de Kodjoboué et les autres. Cette dissemblance pourrait s'expliquer par le fait que Kodjoboué est située sur un affluent de Comoé qui est presque stagnant. Par ailleurs, cette station diffère des autres par de faibles valeurs de conductivité et de valeurs élevées de transparence. 


\section{Conclusion}

Cette étude a permis de recenser 67 espèces de poisson reparties en 11 ordres, 30 familles et dont 16 sont signalées pour la première fois dans le bassin du fleuve Comoé. En ce qui concerne la distribution de poissons, les indices de Shannon et d'équitabilité indiquent des peuplements peu diversifiés et régulièrement repartis. De plus, il n'y a pas de différence significative entre les stations à toutes les saisons. Au niveau des paramètres physiques et chimiques, seule la température présente des variations significatives. Au niveau spatial, seules la transparence et la conductivité présentent des variations. Par ailleurs, la conductivité, la transparence, le $\mathrm{pH}$ et l'oxygène dissous apparaissent les plus discriminants de la répartition des taxons. $\mathrm{Vu}$ l'absence de tout suivi et contrôle, le type de gestion proposée par l'Approche Ecosystémique des Pêches (AEP) devrait être appliqué si l'on veut minimiser la pression qui est exercée.

\section{Remerciements}

Ce travail a été réalisé dans le cadre d'un projet de recherche intitulé « Approche écosystémique de la pêche comme moyen d'exploitation durable des ressources halieutiques dans la zone humide de Grand-Bassam ». Ce dernier a bénéficié des financements du Programme d'Appui Stratégique à la Recherche Scientifique (PASRES) (Côte d'Ivoire). Les auteurs remercient les promoteurs du projet ainsi que toute l'équipe du Laboratoire d'Environnement et de Biologie Aquatique de l'université Nangui Abrogoua et de l'Université Jean Lorougnon Guédé pour leur assistance.

\section{References:}

1. Ahouansou, M. S. (2011). Diversité et exploitation des poissons de la rivière Pendjari (Benin, Afrique de l'Ouest). Thèse de doctorat de l'Université 'Abomey-Calavy, Benin, 216p.

2. Arnason, R. (1997). Fishing: General profile. In Encyclopedia of Occupational Health and Safety, Vol. 03, ILO publications. Pp. 662664

3. Caillart, B. \& Morize, E. (1991). Potentiel halieutique d'un atoll de l'archipel des Tuamotu (Polynésie française) : méthodologie d'étude. In : la recherche face à la pêche artisanale, Symp. Int. ORSTOM IFREMER, Montpellier France, 3-7 juillet 1989, (J-R.) Durand, (1.) Lemoalle et (1.) Weber (eds). Paris, ORSTOM, 1991, tome 1 : pp 325332.

4. Charles-Dominique, E. (2008). Quelle connaissance pour quel développement des pêches artisanales ? Une base de connaissance (ontologie CRM CIDOC) pour l'Afrique de l'Ouest. Rapport XXIV ème Journées du Développement de l'Association Tiers-Monde 
ECONOMIE DE LA CONNAISSANCE ET DEVELOPPEMENT SAINT LOUIS DU SENEGAL, 10p.

5. Chikou, A., Lalèyè, P. A., Raemakers, V., Vandewalle, P. \& Philippart, J-C. (2008). Etude de l'âge et de la croissance chez Clarias gariepinus (Pisces, Clariidae) dans le delta de l'Ouémé au Benin (Afrique de l'Ouest). Int. J. Biol. Chem. Sci. 2(2) : 157-167.

6. Daget, J. \& Iltis, A. (1965). Poissons de Côte d'Ivoire (eaux douces et saumâtres). Mémoires de l'Institut Fondamental de l'Afrique Noire 74, $385 \mathrm{p}$.

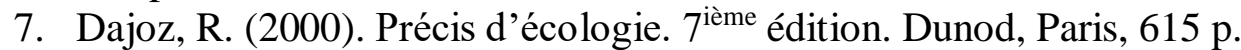

8. Diouf, P. S. (1994). Techniques d'échantillonnage des peuplements de poissons. Document CRO de Dakar Thiaroye, 9 p.

9. Durand, J.-R., \& Chantraine, J.-M. (1982). L'environnement climatique des lagunes ivoiriennes. Rev. Hydrobiol. trop. 15 (2) : 85113.

10. Edia, O. E., 2008. Diversité taxonomique et structure des peuplements de l'entomofaune des rivières côtières Soumié, Eholié, Ehania, Noé (Sud-Est, Côte d'Ivoire). Thèse de doctorat, Université AboboAdjamé, Abidjan, Côte d'Ivoire, 153 p.

11. Edia, O. E., Castella, E., Konan, M. K., Gattolliat, J-L. \& Ouattara, A. (2016). Diversity, distribution and habitat requirements of aquatic insect communities in tropical mountain streams (South-eastern Guinea, West Africa). Ann. Limnol. Int. J. Lim., 52: 285-300.

12. Gascuel, D. (2009). L'approche écosystémique des pêches, une condition pour l'exploitation durable des océans, Pour, 3/2009 $\left(\mathrm{N}^{\circ}\right.$ 202-203), p. 199-206.

13. Gooré Bi, G. (1998). Contribution à l'étude des crevettes d'eau douce de Côte d'Ivoire : systématique, biologie et analyse socio-économique de la pêche de Macrobrachium vollenhovenii (Herklots 1857) et de $M$. macrobrachion (Herklots 1851) (Crustacae Decapoda, Palaemonidae) du bassin de la Bia. Thèse de Doctorat. Université de Cocody, Abidjan (Côte d'Ivoire), $145 \mathrm{p}$.

14. Gordon, N. D., McMahon, T. A. \& Finlayson, B. L. (1994). Stream Hydrology, an Introduction for Ecologists, Wiley \& Sons, New York, $526 \mathrm{p}$.

15. Gourène, G., N'goran, J. Y., Traoré, K. S. \& Ouattara, A. (2002). Caractérisation de l'environnement physico-chimique des poissons d'un bassin côtier Ouest africain (Agnébi : Côte d'Ivoire). Agronomie Africaine, 14 : 139-151.

16. Iltis, A. \& Lévêque, C., 1982. Caractéristiques physico-chimiques des rivières de Côte d'Ivoire. Revue d'Hydrobiologie Tropicale, 15 : 115150. 
17. Issola, Y., Kouassi, A. M, Dongui, B. K. \& Biémi, J. (2008). Caractéristiques physico chimiques d'une lagune côtière tropicale: lagune de Fresco (Côte d'Ivoire). Afrique SCIENCE 04(3) : 368-393.

18. Jaccard, P. (1908). Nouvelles recherches sur la distribution florale. Bulletin de la société. Vaudoise des Sciences Naturelles 44 : 223-270.

19. Jensen, K. W. (1957). Determination of age and growth of Tilapia nilotica (L), T. galilea (Act.), T. zillii (Gerv.) and Lates niloticus C. et V. by means of their scales. K. Nor. Vidensk. Selsk. Forh. 30(24): 150157.

20. Keumean, K. N., Bamba, S. B., Soro, G., Metongo, B. S., Soro, N., \& Biémi, J. (2013). Evolution spatio-temporelle de la qualité physicochimique de l'eau de l'estuaire du fleuve Comoé (Sud-est de la Côte d'ivoire). Int. J. Biol. Chem. Sci, 7(4) : 1752-1766.

21. Konan, K. F. (2008). Composition, structure et déterminisme de la diversité ichtyologique des rivières côtières du Sud-Est de la Côte d'Ivoire (Soumié-Eholié-Ehania-Noé). Thèse de doctorat, Université Abobo-Adjamé, Abidjan, Côte d'Ivoire, 169 p.

22. Konan, K. S., Aka, M. K., Amma, A. A., Dongui, B. K., \& Dago, G. (2008). Variations saisonnières des paramètres abiotiques des eaux d'une lagune tropicale : la lagune de Grand-Lahou, Côte d'Ivoire. European Journal of Scientific research $21 \mathrm{~N}^{\circ} 3, \mathrm{n}^{\circ}$ ISSN 1450-216X.

23. Lalèyè, P., Chikou, A., Philippart, J. C., Teugels, G. G. \& Vandewalle, P. (2004). Etude de la diversité du bassin du fleuve Ouémé au Benin (Afrique de l'Ouest). Cybium 28 (4): 329-339.

24. Lemoalle, J. (1999). La diversité des milieux aquatiques. In Lévêque C. \& Paugy D. (Eds.) : Les poissons des eaux continentales africaines. Diversité, écologie, utilisation par l'homme. Edition IRD, Paris : 11 30.

25. Lévêque, C. (1995). L'habitat : être au bon endroit au bon moment? Bulletin Français de Pêche et de Pisciculture., 337-339 : 9-20.

26. Marcon, E. (2015). Mesures de la biodiversité. Document Unité Mixte de Recherche / Ecologie des Forêts de Guyane, version 2015, 178 p.

27. Matthes, H. (1964). Les poissons du lac Tumba et de la région d'Ikela : Etude systématique et écologique. Annales du Musée royal de l'Afrique centrale 126, $204 \mathrm{p}$.

28. Millet, B. (1984). Hydrologie et hydrochimie d'un milieu lagunaire tropical : Le lac Togo, Thèse de Doctorat, 230 p. Paris : Edition de l'ORSTOM.

29. Moreau, J., Jubb, R. A. \& Arrignon, J. (1988). Les introductions d'espèces étrangères dans les eaux continentales africaines. Intérêts et limites. In Lévêque C., Bruton M. N. et Ssentongo G. W., éd. : 
Biologie et écologie des poissons d'eaux douces africaines. Paris, ORSTOM, Travaux et documents, $216: \mathrm{p}$ 195-245.

30. Niamien-Ebrottié, J. E. (2010). Composition et distribution spatiale et saisonnière des peuplements d'algues de quatre rivières du Sud-Est de la Côte d'Ivoire (Soumié, Éholié, Éhania et Noé), Thèse de doctorat de l'Université Abobo-Adjamé, Abidjan, Côte d'Ivoire, 118 p.

31. Okasanen, Y. F., Blanchet, G., Kindt, Legendre, P., Minchin, P. R., O'hara, R. B., Gavin, L., Simpson, Solymon, P., Stevens, M. H. H. \& Wagner, H. (2013). "Vegan: Community Ecology package", Librairie $\mathrm{R}$ en ligne. Url : http//CRAN.R-project.org/package=vegan.

32. Paillex, A., Castella, E. \& Carron, G. (2007). Aquatic macroinvertebrate response along a gradient of lateral connectivity in river floodplain channels. J. N. Am. Benthol. Soc., 26(4) : 779-796.

33. Paugy, D., Benech, V. \& Diouf, P.S. (1994). Faute ichtyologique des eaux douces de l'Afrique de l'Ouest in Diversité biologique des poissons des eaux douces et saumâtres d'Afrique. G. G. TEUGELS, J. F. GUEGAN \& J. J. ALBARET (Eds.) MRAC-Tervuren-Belgique. Ann. Sc. Zool. (275) : 5-47.

34. Paugy, D., Lévêque, C. \& Teugels, G. G. (2003a). Poissons d'eaux douces et saumâtres de l'Afrique de l'Ouest. Collection faune et flore tropicales, $\mathrm{n}^{\circ}$ 40, MARC/MNHN/IRD, tome 1, Paris, $457 \mathrm{p}$.

35. Paugy, D., Lévêque, C. \& Teugels, G. G. (2003b). Poissons d'eaux douces et saumâtres de l'Afrique de l'Ouest. Collection faune et flore tropicales, $\mathrm{n}^{\circ}$ 40, MARC/MNHN/IRD, tome 2, Paris, $815 \mathrm{p}$.

36. Paugy, D., Lévêque, C. \& Mouas, I. (2011). Poissons d'Afrique et peuples de l'eau. Editions IRD Marseille, France, 313 p.

37. Pauly, D. (1980). On the interrelationships between natural mortality, growth parameters and mean environmental temperature in 175 fish stocks. J. Cons. Int. Explor. Mar., 39 (2): p 175-192.

38. Pielou, E. C. (1966). The measurement of diversity in different types of biological collections. Journal of Theoretical Biology 13 : 131-144.

39. Ramsar, Iran. (1971). Convention relative aux zones humides d'importance internationale particulièrement comme habitats des oiseaux d'eau, $6 \mathrm{p}$.

40. Ramsar (2005). Fiche descriptive sur les zones humides Ramsar (FDR). Catégories approuvées dans la Recommandation 4.7 modifiée par la Résolution VIII.13 de la Conférences des parties contractantes, $17 \mathrm{p}$.

41. Rios, S. L. \& Bailey, R. C. (2006). Relationships between riparian vegetation and stream benthic communities at three spatial scales. Hydrobiologia, $553: 153-160$. 
42. Sankaré, Y. \& Etien, N. (1991). Analyse des effets de l'ouverture du chenal de Grand-

43. Bassam (estuaire du fleuve Comoé, Lagune Ebrié) sur le macrofaune benthique lagunaire. Journal Ivoirien d'Océanologie et Limnologie, $1(2): 81-90$.

44. Secrétariat de la Convention de Ramsar. (2013). Le Manuel de la Convention de Ramsar : Guide de la Convention sur les zones humides (Ramsar, Iran, 1971), 6e édition. Secrétariat de la Convention de Ramsar, Gland, Suisse.

45. Shannon, C. E. (1948). A Mathematical Theory of Communication. The Bell System Technical Journal 27: 379-423 et 623-656.

46. Shannon, C. E. \& Weaver, W. (1963). The Mathematical Theory of Communication. University of Illinois Press (cf. p. 44, 57).

47. Somé, K., Dembélé, Y., Somé, L \& Millogo, R. J. (2008). Pollution agricole des eaux dans le bassin du Nakanbé : cas des réservoirs de Loumbila et de Mogtédo au Burkina Faso. Sud Sci. Technolo., 16.

48. Statsoft Inc (2005). STATISTICA for windows version 7.1. Data analysis software system. http://www.statsoft.com.

49. Teugels, G. G., Lévêque, C., Paugy, D. \& Traoré, K. (1988). État des connaissances sur la faune ichtyologique des bassins côtiers de Côte d'Ivoire et de l'Ouest du Ghana. Revue d'Hydrobiologie Tropicale 21 (3) : 221-237.

50. Traoré, K. (1996). Etat de connaissance sur les pêcheries continentales ivoiriennes. Rapport de consultation, Projet FAO TPC/IVC/4553, IDESSA, Bouaké, Côte d'Ivoire, $131 \mathrm{p}$.

51. Vanga, A. F., Gourène, G. \& Ouattara, M. (2002). Impact de la pêche sur la disponibilité en poissons dans les lacs d'Ayamé et de Buyo (Côte d'Ivoire). Arch. Sc. Cent. Océanol. Abidjan, XVII (2): 1-12.

52. Yao, S. S., Kouamelan, E. P., Koné, T., N’douba, V., Gooré Bi, G., Ollevier, F. \& Thys Van Den Audenaerde, D. F. E. (2005). Fish communities along environmental gradients within the Comoé River basin, Côte d'Ivoire. African Journal of Aquatic Science 30 (2) : 185194.

53. Yao, S.S. (2006). Etude de la diversité biologique et de l'écologie alimentaire de l'ichtyofaune d'un hydrosystème ouest africain : cas du bassin de la Comoé (Côte d'Ivoire). Thèse de Doctorat, Université Cocody-Abidjan, Côte d'Ivoire, 194 p. 